\title{
ADVANCED COMPOSITES REPAIR ANALYSIS TOOL (ACRAT)
}

\author{
Thomas E. Mack \\ The MacNeal-Schwendler Corporation \\ 2975 Redhill Ave. \\ Costa Mesa, CA 92626 \\ James Y. Song \\ USAF Advanced Composites Program Office \\ WL/MLS-OL \\ 5225 Bailey Loop, Bldg. 243E \\ McClellan AFB, CA 95652-2510
}

\begin{abstract}
The Advanced Composites Repair Analysis Tool (ACRAT) has been under development for the USAF Advanced Composites Program Office under an Ogden ALC Design Engineering Program (DEP) Contractual Engineering Task (CET) Order. ACRAT is an integrated prototype software system consisting of commercial-off-the-shelf (COTS) and public domain CAE simulation codes and customized databases. The objective has been to develop Beta versions of this computer aided composite repair design and assessment engineering tool following the provisions and procedures of the ASTM Standard E 1340-90 (Reference 1). The standard guide produces working models early in the development cycle, which allows users and developers to learn functional requirements and appropriate system design details by actually interacting with a series of prototypes. Two Beta versions, of a planned six Beta cycle development effort (threeyear program), have been completed to-date. The proposed ACRAT solution will reduce the Air Force's dependence on airframe manufactures for engineering assistance, reduce component scrap rates, reduce aircraft down-time while repairs are being designed or because spare parts are not available, and reduce the manpower spent removing and reinstalling aircraft parts that could have been repaired on-aircraft.

ACRAT's software system design emphasis has been focused on standardized methods of electronic data modeling and exchange; a UNIX-less process management environment which insures geometric configuration management and material release control; integration of COTS and/or public domain software wherever possible to reduce the cost of development while maximizing system functionality and reducing future costs of ownership (upgrading/porting supportability); and a single user friendly graphical user interface (GUI) capable of supporting a wide range of perspective users (field technician or expert CAE/composites engineer).

Four (4) customized database schemas (M\&P, Aircraft Design, Composite Repair, Component Test) were designed and sufficiently populated to evaluate each data model's ability to meet specific ACRAT system requirements. These prototype databases, coupled with the ACRAT Executive Control System (ECS) database, represent the foundation upon which the fully self contained ACRAT software system has been built.
\end{abstract}




\section{INTRODUCTION}

As advanced composite materials replace metals in aircraft structural components, the task of aircraft maintenance becomes significantly more difficult. Composites exhibit unique and complex material behavior that increases the difficulty of designing and analytically assessing valid structural repairs. Field and depot engineers, responsible for damage assessment and repair designs for today's advanced composite material aircraft components, currently do not have sufficient reliable software tools to design high confidence repairs. As a result, repairable parts may be scrapped because engineers are unable to efficiently assess their damage and define repairs that have been or can be analytically correlated with actual repaired component test results. It was determined in Reference 2 that more sophisticated analytical tools are needed by Air Force engineers to increase their productivity while designing and assessing composite structural repairs. It was also determined that these tools must be supported by a materials and processes database, an aircraft design database, a repair database, and a component test database. It was further established that fully integrated and seamless exchange interfaces to a variety of structural analysis programs, including finite element procedures, are required so that engineers can more quickly and reliably gather input data required to simulate (model) the damaged and repaired structure, execute appropriate analysis code(s), review the analysis results, and then adjust the designs to achieve an optimum repair design configuration.

The principal objective of the ACRAT development effort has been to provide a robust integrated CAE and database software system capable of assisting both engineers and technicians in determining reliable structural repairs for advanced composite aircraft structures. The primary goals of ACRAT's future use include improved reliability of aircraft composite structural repairs, a medium to train Air Force structural engineers in designing valid repairs and field technicians in performing these repairs, and the insertion of repair technology throughout the Air Force resulting in increased maintainability of composite aircraft structures.

\section{PROBLEM DEFINITION}

A comprehensive composite repair requirements definition study was completed during a Phase I ACRAT development effort. A nation wide survey, a detailed literature search, and actual on-site visits to both government and industry facilities were conducted to establish the state-ofthe-art in composite repair design, test and analytical assessment. The results of this study have been documented in Reference 2, and are summarized briefly below:

- The basic physics of composite material behavior are difficult to model and analytically assess. Even more difficult are composite assessments that involve discontinuous structural interfaces, like those typical of "unscheduled" joints required to repair inservice composite component damage. Therefore, the design and analytical assessment of valid composite repairs requires either a skilled composites engineer with advanced CAE tools or a comprehensive database of repair data, guidelines and examples, or both. Figure 1a illustrates typical in-plane bolted repair failure mode sensitivities to varying composite laminate architectures and geometric attachment details. This type of test data, correlated with analytical simulation predictions, are critical to bolted repair design assessment functionality within the proposed ACRAT software system. One can postulate similar laminate composite architecture and geometric detail influences on through-the-thickness bonded repair failure modes and load levels, as depicted in Figure 1b. Because of the costs which would be associated with testing a nearly endless list composite repair details, analytical simulations which have been validated by correlations with actual test results are critical to the ACRAT solution. 
- A significant number of publications exist that discuss the development, testing, analysis and application of composite materials (i.e., Applied Science and Technology Index search turned up more than 1500 separate articles over the past 10 years), but only a few (18 of the aforementioned 1500) discuss repairing composites. These 18 articles on repair are summarized in Appendix A of Reference 2.

- A limited number of special purpose public domain analysis codes are used for both metal and composite structure repair assessments:

- BREPAIR, CREPAIR, BJSFM, and JOINTGAP for modeling bolted repairs

- PGLUE (Reference 4), A4EI (Reference 5), A4EJ, A4EK, DBLLAP3, BOND3/4 for modeling bonded repairs

These 1D and 2D closed form and first order FEA codes have limited use for reliable detailed repair assessments. They are basically used to define first cut repairs that must then be tested for validation. Limited test/analysis correlations have been completed with these tools and little documentation or user friendly GUI environments exist for these tools. The ACRAT solution must be sufficiently open in its software architecture to accommodate the integration of these types of industry accepted software products including the development of graphical interfaces consistent with the overall ACRAT GUI environment.

- The only analytical simulation tools determined to be sufficiently capable of modeling and assessing detailed composite component structural repair details are today's leading commercial FEM and FEA CAE software products. The ACRAT Phase I study identified a good-news, bad-news scenario, i.e., FEA solution results showed excellent correlation with test demonstrated repair joint failures, but a detailed 3D solid model idealization at the ply level was required to accurately predict repair responses that have a strong dependence on inter-ply and bondline failure modes (bondline fracture, ply delamination, etc.). Although FEA tools and/or methods that provide 2D Lamination Theory (LT) or smeared composite property approximations can provide sufficient farfield static and dynamic structural component assessment results, their basic assumptions eliminate the ability to predict actual demonstrated failure modes (i.e., strain components that induce delamination in laminates (cross-ply tension coupled with interlaminar shear) are ignored in 2D LT formulations while through-the-thickness smeared composite stiffnesses also smear the critical bondline strain distribution, resulting in an unconservative prediction of joint failure loads). Figure 2 illustrates the need for ply level FEA idealizations, and typical test data and analytical simulation results that reinforce this observation.

- The only ACRAT development tasks more difficult to accomplish than those associated with accurately simulating the basic physics of composite repair assessment are those associated with defining the data modeling (customized database schemas) and fully electronic seamless data exchange procedures required to support the definition of valid repair designs for complex structural components and assemblies typical of today's state-of-the-art advanced composite aircraft (i.e., C-17,. B-2, F-22, etc.). The data provided to support aircraft logistics tasks is extremely diverse (properties, processes, geometry, loads, design allowables, etc.), voluminous in its content, and in many cases available only in non-electronic format (i.e., drawings, reports (design/stress), etc.). Similar to building a home on an unsound foundation, an ACRAT software solution not built on a sound data model foundation could not support the repair design and 
assessment software loads placed upon it. Significant effort must be placed on the definition of data model schemas and data exchange procedures, critical to the ACRAT solution. Figure 3 summarizes, pictorially, the relation levels defined for each of ACRAT's four (4) required database schemas and typical attribute designations and tables required within these relational levels.

- A significant amount of data, required within the ACRAT process, must be stored, accessed and used in a variety of native formats (can not be populated within the four customized database schemas). The following is a partial list of these data types;

- $\quad$ CAE pre- and post-processing FEM/FEA databases

- Image files that provide process instruction, structural details, assessment results, etc.

- CAD databases

- CAE FEA solution results files

- Text files (lofts tables, laminate stacking tables, etc.)

- Customized spreadsheet files

- Etc.

To store and manage this diverse list of data types, an Executive Control System (ECS) must be defined with its own database structure, as depicted in Figure 4. Besides providing structure to the ACRAT solution, the ECS must provide a basis for an environment that could support a UNIX-less user interface. This inturn would provide a wide range of user skill-level access to the ACRAT System's diverse functionality.

Given this formidable list of issues and system requirements, the ACRAT Software System design was defined and rapid prototyping development cycles initiated under a Phase II development program. The contents of this paper present the progress to date in this rapid prototyping development effort.

\section{SOFTWARE SYSTEM DESIGN CRITERIA}

The criteria which established the guidelines for the ACRAT Software System development effort included; a process for software product development; an emphasis on the use of standardized methods of electronic data modeling and exchange procedures; a recommendation for the use of COTS and/or public domain software products, where ever possible, allowing ACRAT's development effort to focus on the overall software system's integration within an open architecture software environment; a requirement for a knowledge-based functionality that supports a variety of user skill levels in the definition of valid composite structure repairs; a CAD/CAE data modeling and exchange scheme that supports automated parameter driven finite element modeling (FEM) and composite material modeling in support of analytical simulation tasks for undamaged, damaged and repaired details; a requirement to provide a UNIX-less user interface environment; a requirement for an image file management scheme that provides native file format storage and access for viewing anywhere within the ACRAT ECS hierarchy. The remaining paragraphs under this subtopic elaborate briefly on each of the above criteria.

When the functional requirements or functional design details for a software system are not well understood, or when experimentation is required to explore some aspects of the proposed system's behavior, the software development guidelines outlined in ASTM's Standard Guide for Rapid Prototyping of Computerized Systems (Reference 1) are recommended. Rapid prototyping procedures produce a series of working models (Beta versions) quickly, and allow the developers and users to "learn" the functional requirements and appropriate system design features necessary to deliver a successful software product. Because of ACRAT's large system size and potential 
integration complexities, this aforementioned software development approach was recommended over more conventional methods, which concentrate on preparing a detailed functional requirements and design document up-front, which is then implemented over a long period of time before the system is delivered and evaluated by the customer.

Computerization can provide a method for the systematic organization of large amounts of diverse and complex data (i.e., hierarchical, relational and object oriented databases), while simultaneously providing for rapid access and seamless exchange of information required concurrently by a variety of disciplines in the process (i.e., CAD designer, CAE simulation, repair technician, test engineer, etc.). A variety of customizable database products and populated databanks are available. Upon review, in ACRAT's Phase I effort, it was determined that four (4) new customized database schemas would be required to support the ACRAT Software System's databank requirements, i.e., materials and processes (M\&P), aircraft design, repair, and component test databases. Once computerized, materials information, CAD and material architecture geometric details, CAE FEM parameters and loads/boundary conditions, and repair guidelines can be more easily integrated into a CAD/CAE analytical simulation process, which can then be updated as required. A significant amount of data required to support the overall repair definition and assessment process must be imported into the system and stored in its native format, i.e., CAD and CAE model databases, image and text files, customized spreadsheet, files, etc.. A robust GUI environment, a UNIX based directory hierarchy and file designation database, and software tools to access and manipulate this native format data must be an integral part of the ACRAT System, infact, the basic software structure upon which the ACRAT solution must be built.

To maximize the extent of ACRAT delivered functionality within the contracted development dollars and to reduce future supportability costs, wherever possible, existing software products and integration tools were to be used. Public domain software codes, critical to the overall ACRAT solution, were to be ported to the selected hardware platform and integrated into the ACRAT System solution. An open architecture software concept would be the only approach capable of this substantial software system implementation.

The Standard Repair Manual (SRM) delivered with each aircraft classification contains a significant knowledge base which must be incorporated within the ACRAT solution. A knowledge-based GUI environment must be provided to establish when an observed damage is within the guidelines of SRM approved procedures. The same knowledge based ACRAT functionality must also provide data from its databases for structural components which were previously assessed for repaired damage outside the SRM limits. Based on the information provided within a damage definition GUI, the Repair database must be queried and recommendations returned based on its conformance with SRM limits or previous similar repairs stored within the ACRAT System.

As stated previously, accurate analytical simulations for both damaged and repaired composite structural details require a 3D idealization at the ply and bonded interface level. Figure 5 shows typical FEA results completed for correlations with actual repaired composite panel test results (Reference 2). This degree of FEM detail established the requirement for parameter driven FEM procedures, typical of those provided by MSC/PATRAN's ${ }^{\circledR}$ (Reference 7) parameter defined session commands and its associated PCL interface programming functionality. If correctly implemented, these modeling procedures would require a minimum definition of panel dimensions, damage size/type/location/composite material architecture (ply stacking sequence and fiber orientations), etc., to affect the automated session driven modeling of the damaged and repaired panel details. Also, if undamaged aircraft component structural details (geometric, material, etc.) and their associated CAE simulation data requirements (i.e., design loads, edge constraints, design allowables, etc.) can be modeled into a series of customized databases (i.e., M\&P, Aircraft Design Details, etc.), then a more generalized parameter driven FEM and FEA functionality could be 
provided within the ACRAT solution. Any procedures that support simplifications of the more complex FEM processes, typical of composite structural component details, greatly improve the ACRAT system's functionality.

The previously discussed ACRAT ECS database, whose basic structure is summarized in Figure 4, must be supported by a user friendly GUI, which provides a common look and feel environment for the overall ACRAT Software System. Similarly, database access tools and data manipulation tools must be provided within this ECS GUI environment. The ECS should be implemented in such a way as to provide a UNIX-less user interface, which would greatly simplify ACRAT's user environment and further provide a wider range of user skill levels capable of accessing the knowledge-based information (i.e., field technician looking up SRM information) or detailed analytical repair design simulations (expert engineer).

The next subtopic presents the specific ACRAT software system design configuration defined for initial Beta version development. Each of the aforementioned system requirements were addressed within these early Beta version implementations.

\section{SOFTWARE SYSTEM DESIGN CONFIGURATION}

In order to rapidly produce a working prototype model of the proposed ACRAT Software System, a series of existing software products and a variety of standardized software integration and GUI environment tools were selected, each based on an individual capability to satisfy specific criteria of the ACRAT composite repair design and assessment process. The following paragraphs summarize the software system design configuration which was defined and implemented into a demonstrable Beta version prototype.

\section{ACRAT ECS Environment}

The ACRAT Executive Control System (ECS) environment is responsible for all higher level process control and data management tasks required by the implemented ACRAT software solution. It directs, manages and deletes all data related to the specific sub-system paths (Databanks, Aircraft, Repair and Test in Figure 4) provided within the basic software structure of the ACRAT System. To provide a consistent user friendly GUI environment, at all levels within the ACRAT solution, the MSC/UIMS PCL form building tool kit was selected for the ECS GUI environment implementation, as illustrated by example in Figure 6. This same GUI form building environment was selected to provide customized MSC/MVISION (Reference 8) spreadsheet formdriven processes, and it is also an integral customization environment delivered as part of the PATRAN open architecture CAE product. As this statement infers, both MVISION and PATRAN were selected as critical software product implementations within the ACRAT Beta version development effort. Their specific roles and implementation details within the ACRAT solution are discussed in more detail later in this sub-topic.

The entire ACRAT process is controlled by the single command form (panel) illustrated in the upper left corner of Figure 6. The user simply selects a particular sub-system and an associated soft-template path within the ECS database hierarchy structure, and then reviews existing ACRAT information or begins a simulation process to model and assess damage and repair details not available in ACRAT's existing data structure or knowledge-base. The ECS keeps track of where the user last exited the system and places him at that same location when he returns. The ECS ensures that the correct customized MVISION spreadsheet and PATRAN database files are accessible at the lowest level of each sub-system hierarchy path (see Figure 4, lower right corner). 
As illustrated in Figure 6, the implemented Beta version ECS provides image and text file viewing tools (public domain XV and workstation text editor, respectively) as well as a comprehensive series of system administration form environments to support the importing, sharing (sub-system to sub-system) and exporting of data, the backup and archiving of inactive hierarchies, the deleting of files and hierarchy levels, etc.. These tools and forms make it possible for the ACRAT's ECS to provide a virtually UNIX-less system administration and simulation process software environment.

The ECS consists of two distinct segments; the ACRAT ECS PCL and the ACRAT server. The roles of these two segments are as follows:

\section{ACRAT ECS PCL}

The code responsible for all sub-system management and user interfacing. It is this part of the ECS where user requests are acted upon. In order to respond to a request, the following steps are typically executed.

- Determine the current sub-system and location in the ACRAT hierarchy

- Determine the requested operation

- Create a command for the ACRAT server

- Send the command to the server and wait for a response

- Take the appropriate action using the information returned by the server

The ECS consists of approximately 31,000 lines of PCL code. The code drives the ACRAT ECS form environment, but is also the same "glue" that connects PATRAN with its analysis modules and user specific customizations.

\section{$\underline{\text { ACRAT Server }}$}

A UNIX server is a separate program that acts as a passive agent, and its only purpose is to respond to requests from other programs (clients). ACRAT is designed to operate in an environment where there will be multiple users for each ACRAT installation. The ACRAT Server has been designed to insure that each client's request is completed before any subsequent requests are honored. This ability is vital in order to guarantee the integrity of the ACRAT server database. The following list defines the specific responsibilities of the ACRAT Server:

- Communication

- Manage ACRAT database (Utilizing Interbase - the same DB library as used by PATRAN

- Manage ACRAT Sub-System files (UNIX file handling)

- Store ACRAT information (level names, file locations, user activity)

The ACRAT Server communicates with the ACRAT PCL using a UNIX facility known as RPC's (Remote Procedure Calls). RPC's are a standard developed by SUN Microsystem that are available on all platforms where PATRAN is available. The ACRAT Server consists of approximately 4,500 lines of ANSI standard C and SQL (with minor extensions). It was written using the Interbase database developer's kit from Borland for SQL code. The server must run on a machine where PATRAN is installed (Beta 2 version limitation only). 


\section{ACRAT MSC/MVISION Database And Customized Spreadsheet Environment}

The MVISION Materials System Builder (MMSB) COTS software product was selected to serve as ACRAT's central electronic databank source for all materials and processes, design, repair, analytical simulation and test data. As discussed previously, and illustrated in Figure 3, four (4) distinct customized database schemas were designed and sufficiently populated with data to serve two separate purposes in ACRAT's Beta cycle development efforts; 1) evaluation of each schema's ability to model the complexities of each data type required within the overall ACRAT system processes; 2) evaluation of all data exchange paradigms implemented within the ACRAT solution.

The computerization of materials and processes data clearly required careful attention to standards to control (material release quality issue) and communicate material property and composite materials' manufacturing process information. The ASTM committee E-49 has sponsored compilation of the ASTM Standards on the building of Materials Databases (Reference 9). The customized M\&P database developed for ACRAT followed the guidelines set forth in this document. All essential attribute types (primary identifiers) designated as a minimum set to uniquely define a particular material (metal, composite, polymer, etc.) or manufacturing process (heat treatment, cure cycle, etc.) have been included within the ACRAT M\&P database schema.

In the "SOURCE_DATA" relation level, at the bottom of each of the four (4) customized MVISION databases (see Figure 3), are several "TABLE" relations that serve very specific tasks within the ACRAT process.

- $\quad$ M\&P Database

CAE SIMULATIONS - Table that contains a complete analytical material property set to support all FEA simulations, i.e., composite, structural, thermal, etc.

- $\quad$ AIRCRAFT Design Database

COMPOSITE $\quad-\quad$ Table that contains a complete description of a particular composite structures layup, i.e., ply designations, thicknesses, orientations, material type, etc.

GEOM_CAE

LOAD_BC

ASSESSMENTS

ALLOWABLES
- Table that contains a complete description of all geometric data, i.e., grids, coordinate frames, etc., necessary to support automated undamaged component FEM constructions.

- Table that contains a complete description of all load and boundary condition information required to support automated CAE FEA assessments.

- Table that describe analytical simulation assessment details, i.e., Linear Static, 2D, 3D, etc.

- Table That Contains All Analytical Allowables Data Used To Establish Failure Indices. 
- $\quad$ REPAIR Database

DAMAGE_DETAIL - Table that contains more specific information about damage details.

REPAIR_DETAIL - Table that contains more specific information about repair details.

KNOWLEDGE $\quad-$ Table that contains damage limits and recommended repair procedures for those conditions defined in a specific aircraft's Structural Repair Manual (SRM) or Technical Orders (TO) document. Used to interact with ACRAT user to define assessment path, i.e., no analysis required or analytical simulation required to define acceptable repair details.

MVISION MMSB provides the basic foundation upon which ACRAT's customized simulation environments and seamless data exchange procedures have been built, as illustrated in Figure 7. A customized MMSB spreadsheet environment (Level 2 in Figure 7a) is invoked at the lowest level of each ACRAT Sub-System hierarchy path ("Apply" button selection in the ECS form, as shown in Figure 6). The initially invoked MMSB session uses a "template" spreadsheet, which has been automatically copied into this lowest directory level. The spreadsheet will be updated as the specific ACRAT session progresses and the user defines his specific database and/or external process needs. If the spreadsheet session requires the execution of a PATRAN process (RPC procedure), a "template" PATRAN database is automatically copied into the lowest directory level before PATRAN is initiated. If the PATRAN RPC function is successfully initiated, a dummy file is placed into this lowest directory so that subsequent ACRAT sessions initiated at this particular hierarchy level can allow the PATRAN process to be invoked by itself (all initial PATRAN sessions must be initiated, however, from a customized spreadsheet environment to take advantage of the process related data gathering procedures).

The MMSB database and spreadsheet product does not support a standalone customizable PCL form driven user environment, typical of that provided by the PATRAN product. The spreadsheet built-in functions and database tools, however, can be used to customize specific tasks, which can include communications with external processes through the Remote Procedure Call (RPC) built-in interface. It is through this RPC interface that a customizable UIMS PCL form environment has been integrated with the MMSB spreadsheet environment (Local Form depiction in Figure 7a and actual ACRAT Damage Definition Form presented in Figure 8). In addition to using the standard RPC spreadsheet interface utilities, the UIMS spreadsheet form environment communicates with all MVISION databases through the Database Programmatic Interface (DPI) tools provided with the MVISION product (Reference 10). This additional software architecture interface provides a customizable SQL interface to the MVISION databases. The DPI features both read and write built-in-utilities.

A customized spreadsheet environment, using a RPC accessed customized UIMS PCL form interface, has also been provided to support both the A4EI and PGLUE bonded repair assessment codes, previously mentioned. These forms provide a user friendly environment for these previously difficult to interface with public domain analysis codes. Similar form driven interfaces are planned for later ACRAT Beta development cycles, for A43J, A4EK, CREPAIR 
codes, and selected CADAS module. This ability to easily provide customized GUI environments and RPC access to public domain software products, within the implemented ACRAT system design is a significant software development functionality critical to the defined ACRAT solution.

In summary, customized spreadsheets (Global Form in Figure 7a) provide a work-space environment to assemble, manipulate, generate, review, question and store database information and/or simulation process generated data. It serves as the global restrictions (assertions) on the assembled database data required to complete a designated process, as depicted schematically in Figure $7 \mathrm{~b}$. The restrictions applied by specific task directed customized spreadsheets, coupled with specific simulation methods accessed from within the spreadsheet environment by RPC interfaces to external processes, generate new data (information) critical to optimized design/analysis iterations and eventually to new data to be stored within the ACRAT ECS and/or MVISION database system. Actually, these final spreadsheet configurations serve as a complete "slice" of all database information required to assess a specific simulation configuration. With only slight modifications, these spreadsheets can quickly evaluate new but only slightly modified, simulation configurations, without the lengthy process of data assembly from the unrestricted MVISION databases.

\section{ACRAT MSC/PATRAN And MSC/PATRAN ADVANCED FEA Environments}

As illustrated in Figures 7a and 8, all ACRAT PATRAN CAE modeling and assessment sessions are initiated from a customized MMSB spreadsheet environment, where all pertinent geometric, materials and FEA assessment details (boundary conditions, loads, allowables, etc.) have been assembled from the appropriate MVISION databases (M\&P, AIRCRAFT DESIGN, Etc.). This assembled information is passed through the RPC interface to FORTRAN code that uses parameterized PATRAN session commands to create a problem specific session file that is then automatically imported into the PATRAN CAE modeling sequence via a customized ACRAT PCL form environment, summarized pictorially in Figure 8.

A "template" PATRAN database, placed automatically at the lowest level in an ACRAT path hierarchy, already had the PATRAN/ADVANCED FEA (Reference 11) analysis preference designated (FEA Code already in place at customer's site). Therefore, upon model completion, the appropriate analysis type (static, modes, etc.) is merely selected and the FEA solution completed automatically. PATRAN's extensive post-processing was used, in concert with a user friendly customized PCL form environment, to display the critical damage and/or repair structural details with their evaluated stress a strain levels. This project specific parameter driven simulation could easily be implemented into any design/assessment paradigm.

\section{SUMMARY AND CONCLUSIONS}

The ACRAT Beta 2 version consists of the collection of specific commercial and public domain software applications required for the evaluation of damaged aircraft composite components and subsequent repair definitions and/or analytical simulations for repairs which fall outside the recommended SRM or TO limits. These products (both COTS and public domain) have been integrated with custom software (C, PCL, FORTRAN, Motif) to simplify the work of repair technician, repair designers and analysts. Specifically, the software elements which comprise the ACRAT Beta 2 software system are as follows:

1. MSC/MVISION Materials System Builder (MMSB) (COTS, V1.2b)

2. MSC/PATRAN (COTS, V1.4)

3. MSC/PATRAN ADVANCED FEA (COTS)

4. MSC/UIMS (COTS)

5. XV (Public Domain) 
6. PGLUE (Public Domain)

7. A4EI (Public Domain)

A basic ground rule for the ACRAT System development was to provide a COTS software framework to minimize the Air Force customer software support requirements, while maximizing the extent of ACRAT's functionality (development dollars spent "gluing" together existing demonstrated software tools, when possible).

The ACRAT software customization provides a UIMS PCL graphical user interface to manage the work session of multiple technicians/designer/engineers in a task/project oriented approach. A typical organization hierarchy is shown in Figure 4. A UIMS form driven interface has also been customized to facilitate communication with each customized MMSB spreadsheet environment. These form driven spreadsheet utilities are accessed through standard Remote Procedure Calls (RPC), and use the MVISION Database Programmatic Interface (DPI) tool kit to complete external accesses (read and write) to MVISION databanks. Finally, PATRAN customized PCL form driven environments have been provided to facilitate automated finite element modeling (FEM) tasks, based on data that has been assembled in a customized MMSB spreadsheet and passed through an RPC function to external codes that create parameterized PATRAN model building session command files.

The ACRAT System constitutes a substantial amount of custom software, which acts as both a "glue" that integrates all ACRAT System levels as well as providing a common look and feel GUI environment. For the delivered ACRAT Beta 2 Version (fully demonstrable on a HP 700 workstation), the approximate inventory of custom software is as follows:

- 47,000 lines of UIMS PCL code

- 30,000 lines of PATRAN PCL code

- 4,500 lines of C, SQL code

- 13,000 lines of FORTRAN code

All Beta cycle demonstrations were successful and both government and industry acceptance was positive. Substantial development must still be completed, however, before a fully operational ACRAT System can be delivered and supported.

\section{ACKNOWLEDGMENTS}

The authors would like to thank the United States Air Force (USAF) for the opportunity to develop and demonstrate such a unique software system, critical to the future supportability requirements of the Nation's present and future composite aircraft. 


\section{REFERENCES}

1. ASTM Standard Guide for Rapid Prototyping of Computerized Systems Current Edition Approved March 30, 1990. Published May 1990.

2. BDM International, Inc., "Advanced Composites Repair Analysis Tool Requirements Definition Report,” BDM/ABQ-93-0092-TR, September 1, 1993.

3. Chang, Scott, Springer, Journal of Composite Materials, November 1982, Volume 16.

4. Structural Joint Analysis, A4EI, AFWAL TR 81-33154, Volumes 1 and II (ADA 117 342, ADB 954-580), WL/FIBRA/ASIAC, Wright-Patterson Air Force Base, Ohio 45433-6553; May 1991.

5. Laminated Composites PGLUE, Derivative of CREPAIR, AFWAL-TR87-3049, Volumes I and II, (ADB 122 724, ADB 121 687), John Fogary, McDonnell Douglas Aircraft Company, St. Louis, MO. Contained within the ORD code, WL-TR-91-3099, 91-3000 (ADB 164 367).

6. Stone, Robert H., "Development of Field Level Repairs for composite Structures," AGARD Conference Proceedings No. 402, April 1984. (DTIC AD \#P005163) Note: Mr. Stone is a Sr. Research Specialist, Lockheed-California company, P.O. Box 551, Burbank, California 91520.

7. MSC/PATRAN® User Manual, Release 1.2, Vols. 1, 2, 3, 4, Publication No. 903000, The MacNeal-Schwendler Corporation, Costa Mesa, CA December 1993.

8. MSC/MVISION Materials System Builder, Users Guide and Reference, Release 1.2, Publication No. 219011, The MacNeal-Schwendler Coporation, Costa Mesa, CA; 15 November, 1993.

9. ASTM Standards on the Building of Materials Databases, First Edition 1993, Publication Code Number (PCN): 03-549093-63, ASTM 1916 Race St., Philadelphia, PA 19103.

10. MSC/MVISION Database Programmatic Interface, Users Guide and Reference, Release 1.2, Publication No. 903042; 15 November, 1993.

11. MSC/PATRAN ADVANCED FEA Application Module User Manual, Publication No. 903031, The MacNeal-Schwendler Corporation, Costa Mesa, CA; September 1992. 